\title{
¿Qué piensan los profesores de química en ejercicio acerca de la resolución de problemas científicos escolares y sobre las competencias de pensamiento científico?*
}

\author{
What do Chemistry Teachers Think about School Scientific Problem Solving and \\ Scientific Thinking Competences? \\ O que os professores de Química em exercício pensam sobre a resolução de problemas \\ científicos escolares e sobre as competências de pensamento cientifico?

\section{Mario Quintanilla, Carol Joglar, Alberto Labarrere, ${ }^{a}$ Cristian Merino, ${ }^{b}$ Luigi Cuellar, ${ }^{c}$ Ismo Koponen. ${ }^{d}$} \\ ${ }^{a}$ Laboratorio de Investigación en Didáctica de las Ciencias Experimentales (GRECIA), \\ Facultad de Educación, Pontificia Universidad Católica de Chile. Av. Vicuña Mackenna 6848, Santiago. \\ Correo electrónico: mquintag@puc.cl \\ ${ }^{\text {b }}$ Laboratorio de Didáctica de la Química, Instituto de Química, Pontificia Universidad Católica de Valparaíso, \\ Chile. Av. Universidad 330, Campus Curauma, Valparaíso. Correo electrónico: cristian.merino@ucv.cl \\ ${ }^{c}$ Departamento de Didáctica, Facultad de Educación, Universidad Católica de Santísima Concepción, Chile. \\ Alonso de Ribera 2850, Campus San Andrés, Concepción. Correo electrónico: 1cuellar@ucsc.cl \\ ${ }^{d}$ Science Department, University of Helsinki, Finlandia. Correo electrónico: ismo.koponen@ @elsinki.fi
}

\begin{abstract}
RESUMEN
El presente trabajo se sitúa desde las orientaciones teóricas y metodológicas derivadas de un programa de investigación iniciado el 2007 (FONDECYT 1070795, 1095149 y 1110598) cuyo propósito ha sido desde entonces identificar y caracterizar las nociones de los profesores de ciencias acerca de la resolución de problemas científicos escolares y las competencias de pensamiento científico, así como la estrategia más adecuada en que ambas dimensiones han de ser abordadas en la enseñanza de la química escolar. Los resultados de primer orden indican que sus nociones sobre competencias de pensamiento científico y resolución de problemas son fragmentadas, poco elaboradas y hasta contradictorias. La explicación plausible a estos resultados, nos parece, es que sus nociones están vinculas a una representación tradicional de la ciencia y de los productos del conocimiento científico, así como a una imagen instrumental simple y dogmática sobre la enseñanza y el aprendizaje de la química.
\end{abstract}

Palabras clave: competencias de pensamiento científico, resolución de problemas, enseñanza de la química.

\begin{abstract}
This work derives from the theoretical and methodological orientations of a research program initiated in 2007 (FONDECYT 1070795, 1095149 and 1110598) whose purpose has been to identify and characterize the notions of science teachers and future teachers of school scientific problem solving and scientific thinking skills, as well as, the best strategy in which science education should be addressed in the school chemistry teaching. First-order results clearly indicated that their notions of scientific thinking skills and problem solving are fragmented, simple, minimally processed and even contradictory. The possible explanation for these results, we believe, is linked to a
\end{abstract}

* Producto científico derivado de los proyectos FONDECYT 1110598 patrocinado por CONICYT-CHILE y el Proyecto AKA04 patrocinado por la Academia de Ciencias de Finlandia. 
Estudios Pedagógicos XL, $\mathrm{N}^{\circ}$ 2: 283-302, 2014

¿QUÉ PIENSAN LOS PROFESORES DE QUÍMICA EN EJERCICIO ACERCA DE LA RESOLUCIÓN DE PROBLEMAS CIENTÍFICOS ESCOLARES Y SOBRE LAS COMPETENCIAS DE PENSAMIENTO CIENTIIFICO?

traditional representation of science and scientific knowledge products, and a simple and dogmatic instrumental image of teaching and learning chemistry.

Key words: scientific thinking competences, problem solving, chemistry education.

\section{RESUMO}

Situa-se nas orientações teóricas e metodológicas derivadas do Programa de Pesquisa FONDECYT 1070795, 1095149 e 1110598 , cujo objetivo foi identificar e caracterizar as noções que possuem os professores de Ciências atuantes em formação sobre a resolução de problemas escolares e as competências do pensamento cientifico, além das estratégias mais adequadas, por meio das quais, essas dimensões podem ser abordadas no ensino da Química escolar. Um questionário tipo Likert contendo 10 dimensões, foi aplicado a uma amostra de professores e analisaram-se 2 dimensões (20 enunciados). Resultados iniciais indicam: as noções sobre competências do pensamento científico e a resolução de problemas são fragmentadas, simples, minimamente elaboradas e, até mesmo, contraditórias. Acredita-se que a possível explicação sobre os resultados esteja ligada à representação tradicional da ciência e dos produtos provenientes do conhecimento científico, assim como de uma imagem instrumental simples e dogmática sobre o ensino e aprendizagem da química.

Palavras chave: competências de pensamento cientifico, resolução de problemas, ensino de química.

\section{INTRODUCCIÓN}

La producción de nuevo conocimiento científico surge a partir de necesidades que se presentan en el mundo cotidiano y que se transforman en situaciones problemáticas específicas que requieren abordajes, planteamientos y modos de solución particulares, para lo cual se ponen en juego las teorías e instrumentos disponibles inherentes a la ciencia. En el complejo y dinámico proceso de solución se producen sistemáticamente variaciones conceptuales a partir de las cuales las diversas teorías, procedimientos, métodos y lenguajes de la ciencia cambian permanentemente.

Esta realidad puede aplicarse también a la enseñanza de las ciencias en todos los niveles educativos y, con matices, en todos los contextos culturales que le dan cabida. Si bien la bibliografía especializada deja ver con suficiente evidencia que, en general, el estudiantado no aprende a cómo enfrentarse a la solución de problemas científicos escolares (PCE), sino que habitualmente memorizan o reproducen fórmulas, definiciones, cálculos, y procedimientos (Bodner \& Herron, 2002; Bodner, 1987).

En la actualidad se revindica la importancia de la solución de problemas como una competencia de pensamiento científico (CPC) imprescindible para el estudiantado de ciencias. En efecto, el aprendizaje acerca del enfrentamiento a la solución de problemas viene siendo, en las últimas décadas, un ámbito de renovado interés en la comunidad internacional de investigación en didáctica de las ciencias. Por tal razón constituye una vía del todo interesante y promisoria para estudiar el nivel de desarrollo alcanzado por el pensamiento y las competencias de los estudiantes y, a la vez, del desarrollo de éstos en el proceso de enseñanza de las ciencias (Chinn \& Malhotra, 2001; Driver et al., 1994; Klahr, 2002).

En consecuencia, y sosteniendo una visión que no necesariamente puede ser compartida, es necesario que los estudiantes, en el proceso de su formación, tengan ocasión de afrontar problemas con la ayuda del profesorado y ensayar así las diferentes estrategias de solución que contribuyen a ampliar sus conocimientos científicos iniciales. Por tanto, compartimos la idea de que para aprender ciencias de verdad hay que problematizar todas las actividades que se plantean en la clase de ciencias (Bloser, 1988; Camacho \& Good, 1989; Quintanilla et al., 2010). 
Quisiéramos insistir en la idea de que resolver problemas científicos auténticos no significa una tarea de 'hacer tradicional' (operacional, instrumental, mecánica, etc.), sino una actividad científica verdadera (Genyea, 1983; Huffman, 1997; Prins et al., 2008), con la cual los estudiantes construyen en un proceso comprensivo e intencionado teóricamente, los nuevos conocimientos que se consideran fundamentales, ya sea para desempeñarse como profesionales o sujetos competentes en el campo de las ciencias, o bien, para ser ciudadanos responsables alfabetizados científicamente (Izquierdo-Aymerich \& Adúriz-Bravo, 2003).

En el ámbito del aula de ciencias de secundaria, diferentes autores (Bodner \& Herron, 2002; Doran, Helgeson \& Kumar, 1995; Garret, 1988) han planteado que para que exista un verdadero problema científico escolar (PCE) que implique un desafío intelectual valioso para que el estudiantado lo enfrente y solucione comprensivamente, es imprescindible que utilice todo su repertorio cognitivo, capital cultural y social, es decir, sus conocimientos conceptuales, procedimentales y actitudinales. Es 'clave' la formulación de una 'buena pregunta' rica en actos metacognitivos que estimule el pensar teórico y promueva la reflexión acerca de la ciencia, su método y naturaleza (Geddis, 1990).

A pesar de las dificultades u obstáculos epistemológicos e inclusive metodológicos de plantear buenas preguntas (relevantes y productivas), que promuevan el aprendizaje de los modelos teóricos de la ciencia que se quieren enseñar en contextos motivadores que despierten su interés, es sobre todo respecto al último punto que la literatura en investigación en didáctica de las ciencias los evidencia como problemáticos, o más bien, poco problemáticos o nada problemáticos. Así, con respecto al planteamiento de un desafío intelectual, podemos decir que la mayoría de los problemas tradicionales del aula son problemas generalmente cerrados o problemas con 'una única respuesta correcta' y en ocasiones una única estrategia de resolución posible (Garret, 1988), con enunciados enormemente simplificados, datos escogidos a priori (generalmente sólo aquellos que se necesitan), consignas de respuesta implícitas y mиy repetitivos con respecto a sus algoritmos de resolución (Gil-Perez et al., 1990).

En el caso de la clase de química, la resolución de problemas parece centrarse en los problemas cuantitativos, con una atención predominante a la nomenclatura, estequiometría y al equilibrio químico. Muchos informes de investigación se centran en el uso de algoritmos, que son las normas que se pueden seguir más o menos automáticamente por sistemas inteligentes de los cuales, en general, se abusa (Bodner, 1987). Nurrenbern \& Pickering (1987) trabajaron en cinco clases diferentes de problemas de química general, en dos instituciones distintas, en las que los estudiantes recibieron exámenes con preguntas tradicionales que podían ser respondidas usando estrategias algorítmicas y preguntas de selección múltiple que requerían la comprensión conceptual de la química para la solución correcta del contenido. Los estudiantes tuvieron mucho más éxito en las respuestas tradicionales que en aquéllas en las que era necesario comprender los conceptos químicos a los que se referían las preguntas.

Para favorecer una "nueva cultura docente de la enseñanza de las ciencias", el discurso científico y las interacciones comunicativas en el aula deberían superar las opciones reduccionistas y dogmáticas del aprendizaje, y promover en los estudiantes competencias y habilidades cognitivo-lingüísticas para facilitar la integración social, estimular el pensamiento creador y la formación de ciudadanos comprometidos con el dinámico engranaje del desarrollo del crecimiento social y económico (Quintanilla, 2006).

Debido a ello, se constata la relevancia de promover competencias de pensamiento científico (CPC) que permitan al alumno afrontar situaciones diversas, sobre la base de un 
cierto dominio de habilidades y recursos que a buen término le faciliten, por ejemplo, leer y escribir comprensivamente, pensar y reflexionar teóricamente, explorar con curiosidad, formular nuevas preguntas y problemas, argumentar y explicar conocimiento científico de una manera ágil y comprensiva. Las CPC representan entonces una combinación valiosa y dinámica de atributos en relación con conocimientos, habilidades, actitudes, valores y responsabilidades ciudadanas que describen e interpretan los resultados de aprendizajes dentro de un programa educativo mucho más amplio y enriquecedor, en el que los estudiantes son capaces de manifestar comprensivamente en diferentes contextos que la ciencia les resulta interesante, valiosa y necesaria.

Así como lo hemos planteado en anteriores trabajos y proyectos (Molina et al., 2012; Quintanilla et al., 2010; Ravanal \& Quintanilla, 2010), la investigación sobre la determinación e identificación de los tipos de competencias científicas a través de las prácticas evaluativas en química y los efectos que tienen sobre el aprendizaje en los alumnos contribuirá, en alguna medida, a explicar y comprender mejor las teorías sobre conocimiento profesional de los profesores de ciencia de las áreas seleccionadas, colaborando en su desarrollo, transformación y optimización permanentes.

De lo referido anteriomente, no hay estudios específicos en Chile que permitan comprender relaciones no sólo descriptivas sino también explicativas en torno a una noción de competencia científica (CC). De aquí que nuestro principal interés radica en identificar y caracterizar inicialmente las nociones teóricas de profesores de química en formación y en ejercicio, con la finalidad de analizar y comprender los problemas que plantean estas nociones en la clase de química. De ahí la valoración del presente trabajo.

\section{TENSIONES SOBRE UNA NOCIÓN COMPLEJA Y POLÉMICA}

Un análisis teórico-empírico de la situación actual en el terreno de la identificación, caracterización, promoción y desarrollo de competencias de pensamiento científico evidencia la carencia de sistemas y situaciones evaluativas que, de manera coherente y sistemática, den cuenta del desarrollo de las competencias, en general, y de pensamiento científico, en particular.

Compartimos la noción de CPC basada en el enfrentamiento a la resolución de problemas para estudiar el pensamiento docente y estudiantil, lo que implica suponer que la realidad, tal y como es, resulta parcialmente determinada para cada sujeto, según su situación social y personal y, como afirma Blumenfeld et al. (1997), dado su mundo de significaciones, esto es, se concibe la realidad como el producto de la construcción que subjetivamente hace de ella el individuo en un espacio colaborativo de significados consensuados simbólicamente. A su vez, esa realidad construida socialmente pasa a tener una cierta "materialidad" o existencia objetiva que se puede visualizar en el desarrollo de determinadas competencias de pensamiento científico en los sujetos individuales y también como sujetos colectivos.

\section{DE LA COMPETENCIA CIENTÍFICA AL SUJETO COMPETENTE EN CIENCIAS}

Existen escasos indicadores y atributos (o no existen en absoluto) de los diferentes planos o niveles del pensamiento donde, teóricamente, podría transitar la noción de 
competencia de pensamiento científico (CPC) o del sujeto competente en ciencias (SCC), durante el complejo proceso de interacción y comunicación en el aula. La tarea o el desafío consiste, entonces, en la elaboración de indicadores y atributos que puedan dar cuenta de este desarrollo y diseñar los instrumentos correspondientes, así como las estrategias de intervención más adecuadas que simultáneamente colaboren con las transformaciones o cambios relevantes irreducibles en el pensamiento del docente de ciencias. Ésta es una tarea que, desde una perspectiva participativa, no puede llevarse a cabo sin la presencia de los profesores como verdaderos protagonistas del cambio en el aula. Se trata, por tanto, de promover y desarrollar una "cultura de la evaluación de competencias" en el profesorado de ciencias, en general, y en el de química, en particular (Quintanilla et al., 2010).

Pese a que las competencias de pensamiento científico (CPC) se han conceptualizado desde las más diversas direcciones epistemológicas y presentan una naturaleza elusiva, nuestro intento se ha dirigido a conformar una representación de ellas que no se limite a determinar la manera de hacer, sino que también ponga de manifiesto las cualidades de lo que hemos denominado sujeto competente (Labarrere, 2012; Quintanilla, 2012). Desde nuestro punto de vista, el sujeto competente en ciencias (SCC) se constituye como actor y agente particular de la acción, ajustada inteligentemente a las circunstancias sociales y culturales, capaz de adaptar o ajustar el contexto a sus necesidades y con un pensamiento capaz de identificar situaciones problémicas (u obstáculos) en la clase de ciencias, y de abordarlas con la conciencia de los recursos propios que constituyen su perfil personal de actuación en la gestión del conocimiento y aprendizaje científicos.

Hecha esta consideración, la CPC emerge como un atributo del sujeto: es competente no la competencia, sino el sujeto, lo cual determina una actuación permanente y sistemáticamente dirigida a poner en evidencia el sustrato personal del actuar competente, así como la valoración y evaluación de la manera en que los distintos sujetos identifican, enfocan y resuelven las situaciones a las que se enfrentan.

En consecuencia, nos parece necesario que los docentes de química (en formación o en ejercicio) sean capaces de diseñar e implementar instrumentos y estrategias coherentes con una noción de competencias de pensamiento científico (CPC) que den cuenta de cómo el estudiantado aprende a comprender y a interpretar la ciencia al enfrentarse a auténticos problemas en el aula o fuera de ella, ya sea con la ayuda del docente o independientemente, y de forma similar a ensayar y proponer estrategias de solución que contribuyan al aprendizaje, y estimulen el desarrollo de la creatividad y el talento del estudiantado.

\section{4. ¿POR QUÉ DESARROLLAR COMPETENCIAS DE PENSAMIENTO CIENTÍFICO?}

Los desafíos y demandas que nos plantea la actual sociedad del conocimiento, requieren de la educación científica, la formación de profesionales cada vez más informados y empoderados del nuevo conocimiento. Esta nueva concepción de la enseñanza de las ciencias, supera mecanismos dogmáticos para el aprendizaje, transciende a una enseñanza que fomenta el desarrollo de habilidades comunicativas, sociales, emotivas y evidentemente cognitivo-lingüísticas, las cuales facilitarían al estudiantado el enfrentamiento creador a situaciones problemáticas de la ciencia escolar.

Al respecto, investigaciones sobre el pensamiento del profesorado, su formación inicial y su práctica profesional demuestran que las CPC desarrolladas en el estudiantado 
de ciencias, están directamente relacionadas con la noción epistemológica o metateórica del profesorado acerca de y sobre las CPC y cómo estos influencian determinadas CPC en sus estudiantes (Quintanilla et al., 2010; Ravanal \& Quintanilla, 2010), obteniendo de esta forma éxito ante actividades desafiantes, que exigen un dominio conceptual elaborado, habilidades, valores y autorregulación de sus aprendizajes, capacidades que se basan en la interacción de aptitudes prácticas y cognitivas que, combinadas, permiten eficacia a la acción (Quintanilla, 2006). Las CPC representan así una combinación compleja y dinámica de atributos en relación a contextos, conocimientos, habilidades, actitudes, valores y responsabilidades, y emergen de un sistema educacional amplío y enriquecedor, que promueve la autonomía, bajo una comprensión de la ciencia como actividad profundamente humana que la hacen más interesante y valiosa para todos quienes la producen y enseñan (Henao \& Stipcich, 2008).

La promoción y el desarrollo de CPC es actualmente un gran desafío, y no ocurre de forma rutinaria, como es el caso de las competencias intelectuales (Zimmerman \& Forlizzi, 2008), por eso las investigaciones actuales en didáctica de las ciencias se dirigen a investigar cómo situaciones de intervención intencionadas de aula o de procesos formativos sistemáticos en este campo pueden posibilitar el desarrollo de estas competencias metacognitivas dentro de cánones rígidos, entretanto sin perder de vista aspectos sociales y humanos (Henao \& Stipcich, 2008).

\section{METODOLOGÍA}

\subsection{IDENTIFICAR Y CARACTERIZAR NOCIONES TEÓRICAS DE CPC}

Nuestro trabajo se formula como un proyecto de formación y desarrollo que pretende producir conocimiento didáctico acerca del pensamiento y conocimiento del profesorado y por el diseño de mediaciones profesionales, que favorecen la apropiación docente de la ciencia escolar concebida como actividad para el enfrentamiento a la solución de problemas científicos, y a la promoción de competencias específicas en el estudiantado. Se ha optado por profesores de química, puesto que en esta área hemos potenciado nuestra formación e investigación en los últimos 6 años. Al respecto se consideraron, en términos generales, los siguientes aspectos operativos de diseño y análisis de campo:

a) Toma de contacto preliminar con colegios interesados en el estudio y con profesores que imparten la asignatura de química en dichas instituciones.

b) Acopio y selección de los antecedentes de los docentes que participan en la investigación.

c) Revisión bibliográfica de investigaciones similares.

d) Diseño y elaboración de un instrumento de evaluación acerca de la imagen de ciencia y su enseñanza.

e) Validación preliminar por expertos del instrumento elaborado y su aplicación a un grupo piloto de 20 profesores.

f) Aplicación del cuestionario a los profesores de química, que constituyen la muestra estudiada (33).

g) Sistematización y categorización de la información acumulada.

h) Análisis y evaluación preliminar de los hallazgos obtenidos. 


\subsection{VALIDACIÓN DEL CUESTIONARIO}

Resulta importante proponer un instrumento de investigación que permita la caracterización de las nociones teóricas de competencias de pensamiento científico, con la finalidad de analizar y comprender los problemas que plantea esta noción en la clase de química, para lo cual se describen brevemente las fases involucradas en la construcción y validación de un cuestionario acerca de la imagen de ciencia de los profesores, y su aplicación preliminar para proponer actividades de reflexión con los docentes que participan en la investigación y, de esta manera, contribuir a mejorar las prácticas de educación científica.

Se ha intentado razonablemente que cada una de las 8 dimensiones del cuestionario y cada uno de los 80 enunciados que componen el instrumento evalúen efectivamente las categorías predeterminadas $\mathrm{y}$, a su vez, que recojan de forma amplia el pensamiento de los profesores de química con respecto a ellas, de manera tal que éste se convierta en una fuente de información importante para la transformación y consolidación de las prácticas de enseñanza de las ciencias. En función de lo anterior, se consultó la bibliografía pertinente y se propusieron las ocho dimensiones preliminares, que fueron caracterizadas y disgregadas metodológicamente en el cuestionario inicial antes citado, del cual se comentará en el siguiente apartado. Posteriormente se administró a 33 profesores de química, de diferentes establecimientos escolares, de la cuidad de Santiago de Chile. En el mismo instrumento, se recogieron otros datos valiosos para el análisis posterior tales como: edad de los docentes; dependencia administrativa del colegio; nivel en que ejercen y desarrollan sus clases; género; años de experiencia profesional, y; universidad de procedencia.

En una primera fase, el instrumento fue sometido a un proceso de determinación de su validez interna por parte de ocho especialistas iberoamericanos en el área de metodología e investigación en didáctica de las ciencias, y a un grupo piloto de 20 profesores de ciencias naturales en ejercicio, en la ciudad de Santiago, que ejercen en colegios municipalizados (10) y particulares pagados (10). Esta primera fase de la investigación se llevó a cabo durante el primer trimestre del año 2006 y el instrumento continua adaptándose sucesivamente hasta su versión final actual. Se buscó que los evaluadores participaran de su valoración en cuanto a la pertinencia de cada uno de los ítems en cada categoría, al igual que en torno a la claridad o no de la formulación y el uso del lenguaje en el que se presentaban las ocho dimensiones (Ravanal \& Quintanilla, 2010).

\subsection{EL CUESTIONARIO}

El instrumento sobre ideas acerca de la imagen de ciencia y educación científica, en esta primera versión, estuvo estructurado por 80 enunciados (E) distribuidos en cada una de las 8 dimensiones metateóricas, formuladas como afirmaciones y organizadas de manera aleatoria, en formato tipo Likert, cada una con cuatro posibilidades de respuesta: Totalmente de acuerdo (TA), Parcialmente de acuerdo (PA), Parcialmente en desacuerdo (PD) y Totalmente en desacuerdo (TD). Se consideró una quinta columna de 'observaciones' con la finalidad de que el profesor en formación o en ejercicio precisara o justificara aspectos relacionados con la comprensión o no de cada uno de los enunciados. La estructura del instrumento definitivo en la que se sistematizan cada uno de los ítems que componen el instrumento, en relación con las dimensiones inicialmente propuestas, identifica 10 categorías por dimensión, según se aprecia en la Tabla 1. 
Estudios Pedagógicos XL, N²: 283-302, 2014

¿QUÉ PIENSAN LOS PROFESORES DE QUÍMICA EN EJERCICIO ACERCA DE LA RESOLUCIÓN DE PROBLEMAS CIENTIIFICOS ESCOLARES Y SOBRE LAS COMPETENCIAS DE PENSAMIENTO CIENTÍFICO?

En este artículo, sometemos a un análisis en profundidad las dos últimas dimensiones metateóricas del instrumento: Resolución de problemas científicos (D7) y Competencias de pensamiento científico (D8). Los ítems específicos se indican en las Tablas 2 y 3, respectivamente.

Tabla 1. Distribución de las diferentes dimensiones y enunciados del cuestionario

\begin{tabular}{|l|c|c|c|c|c|c|c|c|c|c|}
\hline \multicolumn{1}{|c|}{ Dimensiones } & \multicolumn{6}{|c|}{ Distribución de los enunciados (E) } \\
\hline D1. Naturaleza de las ciencias & 5 & 7 & 22 & 27 & 40 & 52 & 56 & 58 & 61 & 66 \\
\hline D2. Enseñanza de las ciencias & 2 & 18 & 21 & 28 & 46 & 59 & 63 & 64 & 71 & 72 \\
\hline D3. Historia de las ciencias & 1 & 14 & 30 & 38 & 53 & 54 & 55 & 68 & 70 & 79 \\
\hline D4. Aprendizaje de las ciencias & 29 & 29 & 36 & 44 & 49 & 50 & 51 & 62 & 65 & 78 \\
\hline D5. Evaluación de aprendizaje de las ciencias & 9 & 12 & 23 & 33 & 35 & 57 & 67 & 69 & 73 & 75 \\
\hline D6. Rol del profesor de ciencias & 3 & 6 & 11 & 17 & 19 & 31 & 37 & 42 & 43 & 45 \\
\hline D7. Resolución de problemas científicos (RPC) & 8 & 10 & 15 & 16 & 20 & 24 & 39 & 60 & 77 & 76 \\
\hline D8. Competencias de pensamiento científico (CPC) & 4 & 13 & 25 & 26 & 32 & 34 & 41 & 47 & 74 & 80 \\
\hline
\end{tabular}

Fuente: elaboración propia.

\section{DISCUSIÓN}

\subsection{DIMENSIÓN 7: RESOLUCIÓN DE PROBLEMAS CIENTÍFICOS (RPC)}

De conformidad con lo establecido en el marco de referencia teórico, la resolución de problemas científicos en el aula implica un conjunto de procesos y actividades a partir de las cuales los estudiantes generan nuevos conocimientos, considerados fundamentales para transitar a sujetos competentes, lo que hace que la actividad de resolución de problemas en el aula sea notoriamente diferente de la simple 'resolución de tareas'. La solución de problemas puede concebirse como toda situación donde el estudiante se ve obligado a realizar una profunda y sostenida actividad cognoscitiva y práctica, es decir, una situación en la que los conocimientos, los procedimientos, etc., resultan insuficientes para alcanzar una finalidad determinada, en virtud de lo cual se generan contradicciones, estados de incertidumbre e insatisfacciones, que mueven al estudiante hacia delante, involucrándolo en un proceso de búsqueda más o menos sistemático y sostenido que, a su vez, conduce y propicia la construcción de conocimientos y el hallazgo de nuevos procedimientos para aprender a pensar con teoría.

La investigación en didáctica de las ciencias naturales nos muestra que, por lo común, los profesores de ciencia en formación y en ejercicio tienen una imagen incompleta, fragmentada y, en ocasiones, superficial, de lo que es un problema científico escolar (PCE), y lo que puede entenderse por solución de problemas científicos (SPC); esta imagen se transfiere casi directamente a los estudiantes y generalmente opera como modelo implícito (tácito) de la interacción profesor-estudiante, en torno a las actividades asociadas con el planteamiento y la solución de problemas científicos (Izquierdo-Aymerich, 2004). 
¿QUÉ PIENSAN LOS PROFESORES DE QUÍMICA EN EJERCICIO ACERCA DE LA RESOLUCIÓN DE PROBLEMAS CIENTÍFICOS ESCOLARES Y SOBRE LAS COMPETENCIAS DE PENSAMIENTO CIENTÍFICO?

Tabla 2. Ítems específicos del cuestionario según dimensión

\begin{tabular}{|c|l|}
\hline \multicolumn{1}{|c|}{ Dimensión 7. Resolución de problemas científicos } \\
\hline Enunciados & \multicolumn{1}{c|}{ Ítems específicos del cuestionario según dimensión } \\
\hline E8 & $\begin{array}{l}\text { Los problemas diseñados para la actividad científica escolar son problemas, sólo } \\
\text { si surgen del mundo real de los estudiantes. }\end{array}$ \\
\hline E10 & $\begin{array}{l}\text { El profesorado de ciencias ha de enseñar a resolver problemas científicos de } \\
\text { manera racional (por ejemplo, el modelo de cambio químico) y razonable (por } \\
\text { ejemplo, la explicación de la combustión de una vela). }\end{array}$ \\
\hline E16 & $\begin{array}{l}\text { El enunciado de leyes, fórmulas y algoritmos de una teoría científica es suficiente } \\
\text { para que los estudiantes aprendan ciencias. }\end{array}$ \\
\hline E20 & $\begin{array}{l}\text { Se debe propiciar la resolución de problemas científicos en distintas asignaturas, } \\
\text { en las que se compartan conceptos teóricos. Por ejemplo, fuerza gravitatoria } \\
\text { (Física); fuerza de disociación iónica (Química). }\end{array}$ \\
\hline E24 & $\begin{array}{l}\text { La resolución de problemas científicos constituye el eje principal de los procesos } \\
\text { de desarrollo del estudiantado en el ámbito de las ciencias. }\end{array}$ \\
\hline E39 & $\begin{array}{l}\text { Es recomendable que el estudiantado se enfrente a problemas científicos } \\
\text { escolares, en los cuales siempre exista una relación teórica entre conceptos. }\end{array}$ \\
\hline E60 & $\begin{array}{l}\text { Un buen problema científico escolar es aquél que siempre conduce a un resultado } \\
\text { numérico. }\end{array}$ \\
\hline E76 & $\begin{array}{l}\text { El profesorado de ciencias debe enseñar a resolver problemas científicos, } \\
\text { entregando las fórmulas y/o algoritmos requeridos por el estudiantado. }\end{array}$ \\
\hline E77 & $\begin{array}{l}\text { No siempre que se enseña un determinado concepto científico se dispone de } \\
\text { equipamiento apropiado, lo que constituye un problema para que los estudiantes } \\
\text { aprendan. }\end{array}$ \\
\hline $\begin{array}{l}\text { Para abordar situaciones problemáticas en la construcción de conocimientos se } \\
\text { ha de considerar el lenguaje cotidiano del estudiantado. }\end{array}$ \\
\hline
\end{tabular}

Fuente: elaboración propia.

\subsection{DIMENSIÓN 8: COMPETENCIAS DE PENSAMIENTO CIENTÍFICO (CPC)}

La competencia es la capacidad de responder con éxito a las exigencias personales y sociales que nos plantea una actividad (científica en este caso) o una tarea cualquiera en el contexto del ejercicio profesional, e implica dimensiones tanto de tipo cognitivo como no cognitivo. Cada CPC se basa en una combinación de aptitudes prácticas y cognitivas, de orden diverso que conjuntamente ponen en funcionamiento la realización eficaz de una acción: conocimientos, motivaciones, valores, actitudes, emociones y otros elementos sociales y culturales. Una competencia es un tipo de conocimiento complejo que siempre se ejerce en un contexto de manera eficiente (Molina et al., 2012; Quintanilla et al., 2010; Quintanilla, 2006). Sin embargo, un punto sustancial planteado por algunos investigadores como Blumenfeld et al. (1997) argumentan que las insuficiencias en la preparación y actualización del profesor de ciencia (en formación y en servicio), en cuanto a 1os contenidos específicos de la asignatura a enseñar, es una primera dificultad que puede limitar gravemente el potencial innovador de cualquier profesor. 
Estudios Pedagógicos XL, $\mathrm{N}^{\circ}$ 2: 283-302, 2014

¿QUÉ PIENSAN LOS PROFESORES DE QUÍMICA EN EJERCICIO ACERCA DE LA RESOLUCIÓN DE PROBLEMAS CIENTÍFICOS ESCOLARES Y SOBRE LAS COMPETENCIAS DE PENSAMIENTO CIENTIIFICO?

Muchos de los esfuerzos por el mejoramiento de la calidad de la educación científica se han centrado en la exploración de las ideas que profesores y estudiantes tienen en diferentes momentos de su desarrollo personal y/o profesional acerca de la naturaleza de la ciencia, sus métodos e intervención en el mundo. Izquierdo-Aymerich \& Adúriz-Bravo (2003) intentan establecer una base epistemológica para la enseñanza de las ciencias a la luz de las nociones contemporáneas sobre la naturaleza de la ciencia y de cómo aprenden los niños y adolescentes, lo cual constituye un aporte muy valioso a la reforma curricular, ya que emergen fundamentos rigurosos y a la vez razonables desde perspectivas diferentes pero complementarias: filosofía e historia de la ciencia, psicología del aprendizaje y didáctica.

Sin embargo, un hecho evidente es que cualquier innovación educativa en este ámbito particular, debe considerar la formación teórica del profesor en formación o en ejercicio. La noción de CPC se transforma en un término sumamente habitual, no por ello menos controvertido en su aceptación y conceptualización. Sin ánimos de profundizar el aspecto conceptual, y solamente con la finalidad de que se comprenda la dirección de los análisis que se realizarán a continuación, se considera pertinente resaltar que la noción de competencia de pensamiento científico se concibe en relación con un estudiante que responde con éxito a las exigencias personales y sociales que plantea una actividad (científica escolar, en este caso), en lo que se considera un cierto grado de dominio conceptual, de habilidades y de recursos, así como una expresión del control del sujeto sobre la situación y sobre sí mismo (autocontrol).

Tabla 3. Ítems específicos del cuestionario según dimensión

\begin{tabular}{|c|c|}
\hline \multicolumn{2}{|r|}{ Dimensión 8: Competencias de pensamiento científico } \\
\hline Enunciados & Ítems específicos del cuestionario según dimensión \\
\hline E4 & $\begin{array}{l}\text { El desarrollo de competencias de pensamiento científico por parte del profesorado } \\
\text { se logra con objetivos e instrucciones claras y precisas. }\end{array}$ \\
\hline E13 & $\begin{array}{l}\text { Un estudiante competente en ciencias genera conclusiones a partir de sus } \\
\text { observaciones sin necesidad de acudir a teorías. }\end{array}$ \\
\hline E25 & $\begin{array}{l}\text { Un estudiante competente en ciencias moviliza conocimientos y habilidades para } \\
\text { manipular eficientemente instrumental científico. }\end{array}$ \\
\hline E26 & $\begin{array}{l}\text { La actividad escolar que desarrolla competencias de pensamiento científico se } \\
\text { centra en la entrega de datos, fórmulas y teorías. }\end{array}$ \\
\hline E32 & $\begin{array}{l}\text { Una competencia de pensamiento científico expresa expectativas valoradas por la } \\
\text { sociedad, el profesorado y el propio sujeto que aprende. }\end{array}$ \\
\hline E34 & $\begin{array}{l}\text { Un estudiante competente en ciencias integra conocimientos, actitudes y valores } \\
\text { de la comunidad científica, en la clase de ciencias. }\end{array}$ \\
\hline E41 & $\begin{array}{l}\text { El desarrollo de habilidades y destrezas que promueve el profesorado contribuye } \\
\text { a las competencias de pensamiento científico para autorregular los aprendizajes. }\end{array}$ \\
\hline E47 & $\begin{array}{l}\text { Un estudiante competente en ciencias reconoce las limitaciones o ventajas de } \\
\text { apoyarse en teorías para explicar un fenómeno. }\end{array}$ \\
\hline E74 & $\begin{array}{l}\text { Un estudiante es competente en ciencias cuando argumenta a partir de la búsqueda } \\
\text { de explicaciones a los posibles resultados. }\end{array}$ \\
\hline E80 & $\begin{array}{l}\text { Las mediciones SIMCE, PSU, PISA, TIMMS reflejan competencias de } \\
\text { pensamiento científico de manera válida y confiable. }\end{array}$ \\
\hline
\end{tabular}

Fuente: elaboración propia. 


\section{RESULTADOS}

A continuación se presentan los resultados que consideramos más relevantes en este análisis preliminar por cada una de las categorías consideradas para ambas dimensiones que, en este caso en particular, fueron aplicadas a profesores de química en ejercicio. Esto es, Resolución de problemas científicos $(10)^{1}$ y Competencias de pensamiento científico (10). Se ha respetado, para este análisis, la distribución original de cada categoría en el cuestionario.

\subsection{DIMESIÓN (D7): RESOLUCIÓN DE PROBLEMAS}

Se individualizan los siguientes "casos" (Tabla 4 y siguientes): E8, E10, E15, E16, E20, E39, E60, E76 y E77. En el análisis de las respuestas a estos enunciados se puede establecer cierta "dispersión" respecto a que se evidencia una división de representaciones de los docentes en torno a la caracterización de las situaciones problemáticas $(5,11,16)$ ligadas al contexto de los estudiantes. No obstante que en las entrevistas analizadas posteriormente (de lo que daremos cuenta en otro artículo) se resalta la importancia de la contextualización del conocimiento, las respuestas ofrecidas en esta oportunidad no guardan relación con ello y no se da cuenta de esta situación en este artículo. Este resultado es interesante porque evidencia que la mayor parte de los profesores de química $(65,6 \%)$ se declara parcialmente de acuerdo o en desacuerdo, lo cual puede ser considerado como evidencia discreta de que requieren mayor especificación para aseverar totalmente la proposición, indicándose, así, una imagen bastante acertada acerca de la naturaleza subjetiva de la solución de problemas y la correspondiente necesidad de contextualizar la afirmación. Los valores anteriores contribuyen a consolidar la idea de que no resulta despreciable la cantidad de profesores que se declaran estar totalmente de acuerdo (TA, $n=11)$.

Al parecer, se comprende el realismo pragmático (Giere, 1992) que caracteriza esta nueva visión de la actividad científica en la escuela o actividad científica escolar (ACE) (Adúriz-Bravo, Merino \& Izquierdo-Aymerich, 2012; Izquierdo-Aymerich \& AdúrizBravo, 2003). No obstante, y en relación con el enunciado 8, podría pensarse que a falta de una gran coherencia exista ambigüedad o poca claridad en la forma de interpretar estas preguntas. Si establecemos que en E8 no se resalta de forma importante la contextualización del conocimiento como factor decisivo a la hora de aproximarse a la ciencia, no queda claro cómo es que frente a este enunciado (E10) haya una gran inclinación hacia la contextualización y aplicación del conocimiento científico en la escuela $(n=23)$. Sin embargo, la interpretación podría ser diferente, puesto que es muy probable que este valor $(69,7 \%)$ esté constituido por la mayoría de los docentes que componen el 63,7\% del E8. Entonces, nos parece que se hace necesario matizar la conclusión, aunque de nuevo no sería despreciable la cantidad de profesores que sí parece inconsecuente en este sentido.

Es casi unánime el hecho de que la algoritmización del conocimiento no es suficiente para una comprensión de los diversos hechos científicos abordados en la sala de clase $(n=28)$. Sin embargo, un grupo de docentes $(n=5)$ considera suficiente el enunciado de fórmulas y leyes como base del aprendizaje de la química. Obsérvese que ningún profesor se pronuncia por la opción Totalmente de acuerdo (Ver Figura 1).

Se omite el E24 por considerarse irrelevante en el análisis de primer orden en este artículo 
Estudios Pedagógicos XL, N²: 283-302, 2014

¿QUÉ PIENSAN LOS PROFESORES DE QUÍMICA EN EJERCICIO ACERCA DE LA RESOLUCIÓN DE PROBLEMAS CIENTÍFICOS ESCOLARES Y SOBRE LAS COMPETENCIAS DE PENSAMIENTO CIENTIIFICO?

El hecho de la inclinación hacia la 'transversalidad' del conocimiento que se aprecia en esta respuesta por un número significativo de docentes $(n=26)$ es ya reconocido por los profesores, lo que pudo confirmarse desde el análisis de las entrevistas y focus group, que no se analizan en este artículo. A pesar de ello, lo relevante es compartir una 'adecuada' caracterización de lo que se está entendiendo por 'resolución de problemas', algo que se ha establecido en el marco teórico de este artículo, a fin de identificar y caracterizar los problemas que se plantean en la sala de clases.

Figura 1. Resumen resultados para la dimensión resolución de problemas

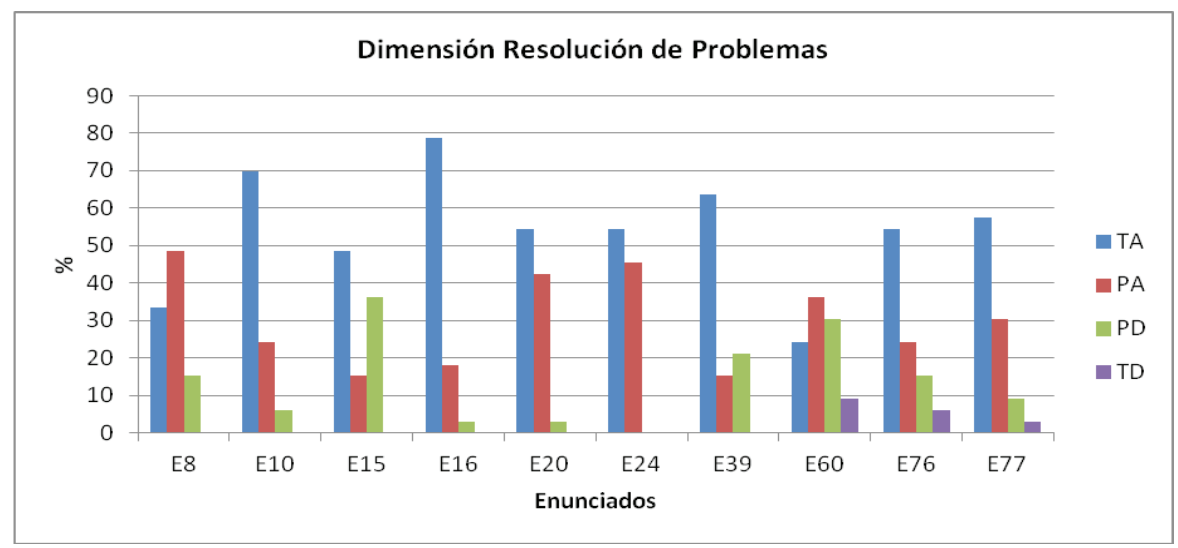

Fuente: elaboración propia.

Éste es un importante factor a considerar: el reconocimiento de la resolución de problemas como una estrategia de aprendizaje, en la que el estudiantado desarrolla pensamiento científico de manera continua, sistemática y permanente, entendido ello como proceso de desarrollo $(n=32)$. De forma similar a lo expuesto frente al enunciado E15, existe ya el reconocimiento de que la simple algoritmización del conocimiento garantiza el aprendizaje de las ciencias $(n=5)$. Podría pensarse, además, cierta diferenciación entre resolución de problemas científicos y desarrollo de 'ejercicios de lápiz y papel'. Los datos evidencian cierta inclinación hacia la pertinencia de entregar las fórmulas y algoritmos a los estudiantes, para que puedan resolver situaciones problemáticas $(n=20)$. Lo anterior permite adelantar dos nuevas conjeturas pues, por un lado, limitaría eventuales procesos de regulación y autorregulación de sus aprendizajes pero, por otra parte, se considera necesaria la aparición del recurso matemático, en este caso, para resolver las situaciones problemáticas. Lo fundamental resulta ser la forma de acceder a ellos por parte de los estudiantes. La respuesta es sumamente interesante, dado que podría estar indicando una valoración y, al mismo tiempo, comprensión insuficiente de la algoritmización y del conocimiento de "las fórmulas", lo cual resulta una instancia necesaria, a la que se puede llegar mediante la "integración" adecuada de la solución de problemas en el proceso de enseñanza, con vista al desarrollo de competencias de pensamiento científico.

Las respuestas obtenidas en la Figura 1 (E76) hacen referencia a las dificultades de no contar con equipamiento especializado para la enseñanza de contenidos científicos $(n=26)$. 
Si bien es cierto que hay inclinaciones hacia esta perspectiva que lo consideran 'en contra' de la enseñanza de la ciencia, bien valdría la pena ahondar en las razones que aducen quienes están a favor y en contra de esta afirmación ( $n=7$ y n=26).

Los profesores de química reconocen la importancia y el valor del lenguaje como instancia de construcción de conocimiento científico escolar $(n=29)$. Esta valoración es de suyo relevante, ya que se da un paso fundamental en la idea de que "enseñar a hablar y a escribir" la ciencia es un factor primordial para promover sujetos competentes, conscientes de la ciencia que aprenden. Nos parece que una buena respuesta (esperable) en este enunciado -implicaría una mayor densidad en Totalmente de acuerdo-; sin embargo, la parcialidad introducida más los que expresan estar Totalmente de acuerdo indican que no existe una comprensión profunda del valor del lenguaje, que implica situarse en el universo representacional del estudiante y, al mismo tiempo, contextualizar las situaciones, nos parece que aquí se muestra con mayor claridad la posible contradicción y el carácter 'superficial' de las respuestas, en el sentido de que no indican una valoración precisa y amplia del elemento subjetivo que implica la solución de problemas. La inferencia, a nuestro juicio, debería hacerse en este sentido.

\subsection{DIMENSIÓN (D8): COMPETENCIAS DE PENSAMIENTO CIENTÍFICO}

A partir de los resultados obtenidos, se podría pensar en que la asociación elaborada por parte de los docentes se considera importante, más allá de la 'rigidez' de una instrucción, diríamos, tradicional, la pertinencia de establecer de forma clara y precisa las condiciones en que el sujeto ha de enfrentarse a las diferentes situaciones problemáticas al abordar un contenido científico $(n=30)$. En una instancia preliminar del análisis -focus group- se pudo establecer que para los profesores de ciencias es fundamental la 'disposición personal para emprender la actividad', ante lo cual podríamos decir que los resultados frente a este enunciado complementarían esta afirmación en el sentido de situar la disposición, en este caso, fuera del individuo, localizándola en las condiciones externas necesarias para la consecución del desarrollo de CPC, lo cual irá perfilándose más claramente en el transcurso de la investigación.

Figura 2. Resumen resultados para la dimensión competencias de pensamiento científico

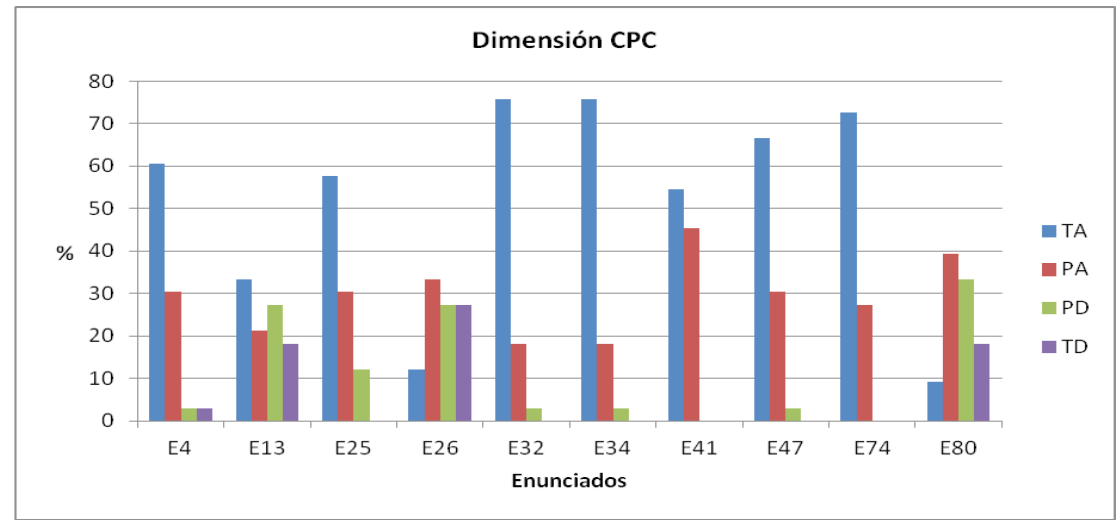

Fuente: elaboración propia. 
Estudios Pedagógicos XL, $\mathrm{N}^{\circ}$ 2: 283-302, 2014

¿QUÉ PIENSAN LOS PROFESORES DE QUÍMICA EN EJERCICIO ACERCA DE LA RESOLUCIÓN DE PROBLEMAS CIENTÍFICOS ESCOLARES Y SOBRE LAS COMPETENCIAS DE PENSAMIENTO CIENTÍFICO?

En cuanto al vínculo entre la observación y la teorización en el desarrollo de competencias científicas se identifican puntos divergentes en el profesorado. Así, el enunciado correspondiente (E13) merece un análisis particular, debido a la heterogeneidad misma de las respuestas obtenidas, las cuales develan multiplicidad de concepciones por parte de los profesores de ciencias. El 54,5\% se inclina relativamente a favor de la afirmación, es decir, en torno a la posibilidad de establecer conclusiones sin tener en cuenta la relación con marcos teóricos establecidos $(\mathrm{n}=18)$. Un aspecto a indagar posteriormente puede ser la posible relación que exista entre estas respuestas y las ofrecidas en torno a la metodología científica, y más específicamente, frente a la adscripción de muchos profesores al 'tradicionalmente' concebido método científico.

Podría decirse que estos resultados confirman lo ya establecido en relación con la importancia que asignan los profesores al componente experimental, inherente a la enseñanza de las ciencias y al desempeño competente de los estudiantes en esta área $(n=29)$.

Éste es otro análisis que nos permite conjeturar en la necesidad de seguir investigando en torno a las concepciones de los profesores acerca de la adquisición de CPC por parte de los estudiantes, ya que, observando las respuestas, al igual que frente al enunciado E13, se ha encontrado una gran diversidad de respuestas. Es así como el 54,6\% (n=18) se manifiesta en desacuerdo a la afirmación, mientras que un $45,4 \%$ se manifiesta a favor de ella $(n=15)$. Al parecer, se cuestiona la 'exclusividad' de la presencia de datos, fórmulas y teorías como garante del desempeño competente del estudiante en el ámbito científico.

Podría pensarse en la inclinación, por parte de los profesores, a considerar que el desempeño competente en ciencias no sólo implica demostraciones de conocimientos, ni planteamientos de respuestas frente a demandas específicas, sino que además se ha de manifestar el involucramiento de la persona en el desarrollo de la CPC. De ser así, se establece, tal y como se hizo en el análisis del focus group, por parte de uno de los investigadores, que estamos frente a representaciones relativamente complejas que implican aspectos afectivos y axiológicos $(n=31)$. Al mismo tiempo, debe notarse que la alta inclusión del sujeto (el estudiante) en esta respuesta, al parecer, contradice respuestas anteriores donde tal inclusión no aparece de manera patente.

A partir de estos análisis descriptivos (E34), puede adelantarse que existe un alto grado de coincidencia a favor de reconocer la actividad de los científicos como actividad humana, constituida por una comunidad de especialistas, ligada a diversos factores contextuales $(\mathrm{n}=31)$. Podríamos interpretar que con lo anterior se privilegian algunas competencias cognitivo-lingüísticas, las cuales se identificarán y caracterizarán posteriormente con el avance de la investigación. Además, se estaría introduciendo un reconocimiento tácito del contexto cultural y la contextualización como componentes obligados del desarrollo de competencias, con lo cual se incluye un aspecto que, como hemos visto, no se ha tomado en consideración de forma consistente en otros enunciados.

Es clara la aceptación (E41), por parte de los profesores de química, de la relación que existe entre el desarrollo de diversas habilidades cognitivo-lingüísticas como promotoras de competencias de pensamiento científico, haciéndose énfasis en la importancia que lo anterior tiene para generar actitudes de anticipación a la acción, cuando los estudiantes abordan el conocimiento científico en el aula $(n=33)$. Los resultados obtenidos frente a este enunciado son complementarios y coherentes con lo establecido en el análisis preliminar. Así como del anterior se puede derivar la importancia de la anticipación a la acción, lo expresado por la mayoría de los profesores frente a este enunciado $(n=32)$ 
permite establecer también cierta claridad respecto a las posibilidades que un estudiante competente en ciencias tendría para optar por una u otra teoría o modelo científico, a fin de afrontar una situación que le resulte problemática. De igual manera, debe prestarse atención a la proporción de docentes que se encuentra a favor de esta proposición, la que se haya en relativa contradicción con la respuesta ofrecida a otras que, bajo diferente formulación, introduce igualmente el factor relativo al manejo de la teoría por parte de los estudiantes.

Frente al encunciado E74 (y al igual que lo expresado en relación con los dos últimos enunciados E41 y E47), es interesante observar cómo los profesores manifiestan la importancia de una de las competencias cognitivo-lingüísticas, argumentar, para enfrentarse a posibles soluciones a situaciones problemáticas específicas $(n=33)$. Se puede inferir a partir de aquí que, para los docentes, un estudiante competente en ciencias no es aquél que llega de manera algorítmica a la consecución de una solución mediante una única e irrevocable vía de acceso al conocimiento.

$\mathrm{Al}$ igual que los hallazgos vinculados con los enunciados E13 y E26, éste es el tercer enunciado (E80) de la dimensión metateórica denominada competencias de pensamiento científico (CPC) que más heterogeneidad presenta en las respuestas por parte de los profesores que hacen parte de la investigación. El 48,5\% de aceptación $(n=16)$ total o parcial, frente al $51,5 \%$ de no aceptación total o parcial $(n=17)$ podría ser considerado un claro indicio de lo polémico que resulta para los docentes el tema de la medición externa, sobre la base de estándares establecidos y distintos de los definidos del proceso que ha vivido el estudiante.

\subsection{DISCUSIÓN Y EVALUACIÓN DE LA DIMENSIÓN (D7): RESOLUCIÓN DE PROBLEMAS (RPC)}

De esta forma, el grupo de 33 profesores de química, de un total de 117 profesores de ciencias, se ha manifestado en torno a esta dimensión de análisis según los resultados consolidados, presentados a continuación y que se resumen en la Tabla 4.

Para el primer caso, podemos observar que, respecto al enunciado E15, el 87,5\% se manifestó en desacuerdo, frente al E39, un 87,5\% se manifestó en desacuerdo, y en relación con el E60, un 40,7\% en desacuerdo, lo cual resulta menos coherente con lo establecido en los dos enunciados anteriores. Además, un 59,4\% se pronunció de acuerdo, lo cual es la base para que podamos debatir sobre posibles contradicciones en el pensamiento de los profesores de química que participaron en esta investigación.

Respecto al enunciado E8, es muy notable que el 30\% de docentes de química esté totalmente de acuerdo, indica un desconocimiento (en el sentido de no conocer y/o no tener en cuenta la dinámica) de la actividad de pensamiento en el curso de la solución de problemas científicos escolares (PCE). Obviamente, no todos los problemas tienen que surgir del mundo real de los estudiantes, pueden también ingresar a su mundo real. Con el alto porcentaje TA $(34,40 \%)$, se reafirma que ven los problemas científicos sólo en su dimensión objetiva (desde la trama disciplinar específica) y no desde su función cognitiva, subjetiva y relativa, con lo cual se puede alterar el trabajo con el pensamiento y con la competencia, en tanto ésta se manifiesta como una componente individual. El porcentaje anteriormente citado parece ser relevante en sí mismo; sin embargo, se matiza al tener en consideración el alto índice que obtiene lo parcial (acuerdo y desacuerdo), con lo cual se podría estar sustentando nuestra interpretación. 
Estudios Pedagógicos XL, $\mathrm{N}^{\circ}$ 2: 283-302, 2014

¿QUÉ PIENSAN LOS PROFESORES DE QUÍMICA EN EJERCICIO ACERCA DE LA RESOLUCIÓN DE PROBLEMAS CIENTIIFICOS ESCOLARES Y SOBRE LAS COMPETENCIAS DE PENSAMIENTO CIENTÍFICO?

Tabla 4. Estadísticas consolidadas de los resultados analizados en la dimensión resolución de problemas científicos

\begin{tabular}{|l|c|c|c|c|c|c|c|c|c|c|}
\hline & P.8 & P.10 & P.15 & P.16 & P.20 & P.24 & P.39 & P.60 & P.76 & P.77 \\
\hline N Valid & 32 & 33 & 33 & 33 & 33 & 33 & 33 & 33 & 33 & 33 \\
$\quad$ Lost & 1 & 0 & 3 & 0 & 0 & 0 & 0 & 0 & 0 & 0 \\
Mean & 3,19 & 3,64 & 3,33 & 3,76 & 3,39 & 3,55 & 3,48 & 2,24 & 1,73 & 3,42 \\
T. Deviation &, 693 &, 603 &, 736 &, 502 &, 556 &, 506 &, 755 &, 936 &, 944 &, 792 \\
Minimum & 2 & 2 & 2 & 2 & 2 & 3 & 2 & 1 & 1 & 1 \\
Máximum & 4 & 4 & 4 & 4 & 4 & 4 & 4 & 4 & 4 & 4 \\
\hline
\end{tabular}

Fuente: elaboración propia.

Frente a los dos primeros enunciados (E8 y E10), es casi unánime el hecho de que la algoritmización (o formalización matemática de la química escolar) del conocimiento no es suficiente para una comprensión de los diversos hechos científicos abordados en la sala de clases, mientras que frente al tercer enunciado (E15) se observa cierta inclinación hacia la pertinencia de entregar las fórmulas y algoritmos a los estudiantes para que puedan resolver situaciones problemáticas tradicionales o no. Del mismo modo, se considera necesario el uso del recurso matemático, en este caso, para resolver las situaciones problemáticas de química, lo que resulta polémico desde concepciones epistemológicas cercanas al modelo cognitivo de la ciencia (Giere, 1992; Izquierdo-Aymerich, 2004), compartidas por nuestro Laboratorio de Investigación y en el cual los modelos teóricos construidos por los estudiantes son paulatinamente más elaborados, coherentes y complejos, y por tanto requieren y, a la vez, propician una base de regulación y autorregulación de sus aprendizajes de manera continua y sistemática (Bodner \& Herron, 2002).

\subsection{DISCUSIÓN Y EVALUACIÓN DE LOS RESULTADOS DIMENSIÓN (D8) COMPETENCIA DE PENSAMIENTO CIENTÍFICO (CPC)}

Tal como se estableció en la dimensión metateórica resolución de problemas científicos (RPC), en la dimensión metateórica referida a las competencias de pensamiento científico $(C P C)$ igualmente interpretamos una tendencia hacia la aceptación de las respuestas (PA y TA) de cada uno de los enunciados que forman parte de esta dimensión y que se pueden visualizar en la Tabla 4. Sin embargo, las respuestas del colectivo en relación con E13 y E26 muestran $(\sigma=1,132$ y $\sigma=1,015)$ una clara heterogeneidad de las respuestas, encontrándose posiciones divergentes, a favor y en contra. Frente a los 10 que conforman esta dimensión metateórica, se destaca la importancia de la orientación como anticipación a la acción, más allá de la rigidez de una instrucción, diríamos tradicional: la necesidad del componente experimental en la clase de ciencias; el involucramiento de la persona en el desarrollo de la CPC específica, incluyendo factores afectivos y axiológicos; reconocimiento del modelo cognitivo de la ciencia, y; la importancia de habilidades cognitivo-lingüísticas como promotoras de competencias de pensamiento científico. Un análisis particular merece las respuestas obtenidas en E13, E26, E47 y E80. 
El enunciado E13 demanda un análisis particular, debido a la heterogeneidad misma de las respuestas obtenidas, las cuales develan multiplicidad de concepciones por parte de los profesores de ciencias, en general, y de los docentes de química, en particular. Es así como el 53,2\% se inclina a favor de la afirmación, es decir, en torno a establecer conclusiones sin tener en cuenta la relación con marcos teóricos científicos establecidos. Los docentes que declaran estar parcialmente de acuerdo (PA) expresan que hace falta teoría, lo mismo que los que están parcialmente en descuerdo (PD); si a ellos se suman los que están totalmente en desacuerdo (TD) se invierte la situación y se obtiene que los profesores valoran una afirmación de esta naturaleza con diferentes matices. Esto es interesante porque podemos oponerlo a lo que realmente se hace o lo que se tiene en cuenta en la práctica del aula de ciencias, lo cual nos lleva al hecho habitualmente constatado de las contradicciones que se tejen entre el pensar, actuar y comunicar la química escolar, aspecto que ha sido constatado y argumentado en investigaciones que se han ocupado del tema en particular (IzquierdoAymerich, Merino y Quintanilla, 2014; Kopoenen \& Pehkonen, 2010; Koponen, 2007).

En cuanto al enunciado E26, al igual que frente al E13, se ha encontrado una gran diversidad de respuestas. Es así como el 54,6\% se manifiesta en desacuerdo con la afirmación, mientras que un $45,4 \%$ se manifiesta a favor de ella. Al parecer se cuestiona la 'exclusividad' de la presencia de datos, fórmulas y teorías como garante del desempeño competente del estudiante en al ámbito científico. Aquí ocurre otro tanto interesante: la suma de TD+PD da cuenta de que los profesores están en contra, o sea, que al parecer conceptualmente hay elementos favorables en este sentido que les llevan a comprender la imagen de la ciencia de esta manera (Toulmin, 1977). Los resultados obtenidos frente a este enunciado son coherentes con lo establecido en el análisis preliminar de aquellos que reconocen la importancia de la anticipación a la acción y de las posibilidades que un estudiante competente en ciencias tendría para optar por una u otra teoría o modelo científico, a fin de afrontar una situación que le resulte problemática para explicar o argumentar sobre la ciencia que aprende. Sin embargo, el alto porcentaje de adscripción a este planteamiento (97\%) resulta contradictorio con lo observado en E13, en el que se destaca la importancia de la observación, más allá de la teorización. Se refiere a la E47 y no es contradictorio, aunque valdría la pena discutir si es válida la comparación; a nuestro entender apuntan a cosas diferentes. Cabe también relevar la heterogeneidad encontrada en las respuestas docentes en lo que se refiere a los enunciados E13, E26 y E80, caracterizados en la dimensión competencias de pensamiento científico.

Tabla 5. Estadísticas consolidadas de los resultados analizados en la dimensión competencias de pensamiento científico

\begin{tabular}{|l|c|c|c|c|c|c|c|c|c|c|}
\hline & P.4 & P.13 & P.25 & P.26 & P.32 & P.34 & P.41 & P.47 & P.74 & P.80 \\
\hline N Valid & 32 & 33 & 33 & 33 & 32 & 33 & 33 & 33 & 33 & 33 \\
\multicolumn{1}{|c|}{ Lost } & 1 & 0 & 0 & 0 & 1 & 1 & 0 & 0 & 0 & 0 \\
Mean & 1,47 & 2,30 & 1,55 & 2,70 & 3,75 & 3,75 & 3,55 & 3,64 & 3,73 & 2,61 \\
T.Deviation &, 718 & 1,132 &, 711 & 1,015 &, 508 &, 508 &, 506 &, 549 &, 452 &, 899 \\
Minimum & 1 & 1 & 1 & 1 & 2 & 2 & 3 & 2 & 3 & 1 \\
Máximum & 4 & 4 & 4 & 4 & 4 & 4 & 4 & 4 & 4 & 4 \\
\hline
\end{tabular}

Fuente: elaboración propia. 
Estudios Pedagógicos XL, $\mathrm{N}^{\circ}$ 2: 283-302, 2014

¿QUÉ PIENSAN LOS PROFESORES DE QUÍMICA EN EJERCICIO ACERCA DE LA RESOLUCIÓN DE PROBLEMAS CIENTÍFICOS ESCOLARES Y SOBRE LAS COMPETENCIAS DE PENSAMIENTO CIENTIIFICO?

\section{CONCLUSIONES E IMPLICACIONES}

Al concluir este artículo podemos plantear básicamente tres ideas que nos parecen irreductibles en función de los hallazgos que hemos presentado: (i) los docentes de química en ejercicio que hemos explorado en este trabajo manifiestan, con matices, diferentes imágenes acerca de la naturaleza de la ciencia, el conocimiento químico enseñado y el aprendizaje; (ii) la visión de los profesores de química frente a las competencias de pensamiento científico (CPC) y a la resolución de problemas científicos (RPC) debe justificarse en gran medida por la formación inicial que han recibido en su proceso profesional, y; finalmente, (iii) resulta evidente que en esta visión de la ciencia construida y enseñada existe un tránsito interesante desde una visión epistemológica categórica de la educación científica, hacia un planteamiento de carácter racionalista moderado (Toulmin, 1972), que actualmente estamos trabajando con 12 profesores de química en ejercicio en nuestro proyecto AKA-04, y otros 70 profesores de química y biología en formación con promisorios resultados a la fecha de edición de este trabajo.

Las observaciones anteriores han sido limitaciones que marcan tanto la evaluación como la formación real de las competencias, es decir, del sujeto competente ante la química y sus problemas. Obviamente al estar ligadas a una insuficiente representación de la competencia de pensamiento científico, han entrañado también una insuficiente o inadecuada evaluación del desarrollo de la competencia y, de hecho, a la formación plena del sujeto competente. La carencia de un cuerpo teórico de conocimientos en didáctica de las ciencias vinculado a las prácticas de enseñanza científica, reconocido por el profesor de química en formación o en ejercicio, es quizá uno de los mayores problemas que evidencian esta situación y que queda de manifiesto a la hora de promover competencias de pensamiento científico de calidad en sus alumnos mediante el enfrentamiento a la resolución de problemas. El proceso de cambio conceptual en ciencia y en enseñanza de las ciencias exige el diseño de una propuesta alternativa que tenga en cuenta los avances de la investigación en didáctica de las ciencias y, al mismo tiempo, le permita al profesor cuestionar el saber y el saberhacer relacionado con su acción docente y profesional cotidiana.

Algunos de los resultados de investigaciones a nivel mundial acerca de la enseñanza de las ciencias y la resolución de problemas muestran que así como los alumnos llegan a clase con ideas personales respecto a los fenómenos, los profesores de ciencias también desarrollan sus propias concepciones frente a la enseñanza, la evaluación y el aprendizaje de los diferentes contenidos específicos. Sin embargo, durante la formación inicial y continua del profesor de ciencias, dichas visiones raramente son tenidas en cuenta y, en consecuencia, no se le prepara para asumir puntos de vista críticos frente al saber, y mucho menos, frente a su actuación docente, lo cual sería posible si durante los cursos de formación inicial y continua hubiese espacio para la reflexión, la interacción social y la regulación de aprendizajes de manera permanente, favoreciendo la comunicación de la ciencia en un espacio en que el lenguaje y el pensamiento teórico tiene una importancia relevante.

En este orden de ideas, la investigación aquí presentada enfatiza la relación entre la metacognición y aprender a enseñar química para el logro de competencias científicas de calidad, pues se argumenta que la metacognición es central para promover los cambios apropiados en el desarrollo del profesor de ciencia, no sólo en lo relativo a sus ideas acerca de la enseñanza y el aprendizaje y los roles de docente y alumno, sino también acerca del contenido erudito de la disciplina, sus habilidades docentes y los fundamentos epistemológicos básicos que debe dominar y que deben ser coherentes con sus prácticas 
evaluativas en el desarrollo de dichas competencias científicas en sus estudiantes. Continuamos trabajando en este ámbito y esperamos tener suficiente evidencia empírica en un próximo artículo para el cual ya estamos sistematizando la respectiva información.

\section{AGRADECIMIENTOS}

Producto científico derivado de los proyectos FONDECYT 1110598 patrocinado por CONICYT - CHILE y el Proyecto AKA-04 patrocinado por la Academia de Ciencias de Finlandia.

\section{REFERENCIAS BIBLIOGRÁFICAS}

Adúriz-Bravo, A., Merino, C. \& Izquierdo-Aymerich, M. (2012). An aproach to the Construction of Chemistry Curricula on the Basis of Structuring Theoretical Fields. Journal of Science Education, vol. 13, 42-44.

Bloser, P. (1988). Teaching Problem Solving-Secondary School Science. Science Education Digest, n. 2, 1-6.

Blumenfeld, P., Marx, R., Patrick, H. \& Krajcik, J. (1997). Teaching for Understanding. In B. Biddle, T. Good \& I. Goodson (Eds.), International Handbook of Teachers and Teaching (Vol. II) (pp. 819-879). Dordrecht: Kluwer Academic Publishers.

Bodner, G. \& Herron, D. (2002). Probem-solving in Chemistry. In J. K. Gilbert (Ed.), Chemical Education: Towards a Research-Based Practice (pp. 235-266). The Netherlands: Kluwer Academic Publisher.

. (1987). The Role of Algorithms in Teaching Problem Solving. Journal of Chemical Education, vol. 64, n. 6, 513-514.

Bodner, G. M. \& Herron, J. D. (2002). Problem-solving in Chemistry. In J. Gilbert, O. de Jong, R. Justi, D. F. Treagust \& J. van Driel (Eds.), Chemical Education: Towards Research-based Practice (pp. 235-266). Dordrecht: Kluwer Academic Publishers.

Camacho, M. \& Good, R. (1989). Problem-solving and Chemical Equilibrium: Successful Versus Unsuccessful Perfomance. Journal of Research in Science Teaching, vol. 26, n. 3, 251-272.

Chinn, C. \& Malhotra, B. A. (2001). Epistemologically Authentic Inquiry in Schools: A Theoretical Framework for Evaluating Inquiry Tasks. Science Education, vol. 86, n. 2, 175-218.

Doran, R. L., Helgeson, S. L. \& Kumar, D. D. (1995). Assessment of Problem Solving in Science. In D. R. Lavoie (Ed.), Toward a Cognitive-science Perspective for Scientific Problem Solving (pp. 112-141). Manhattan, Kansas: NARST.

Driver, R., Leach, J., Scott, P. \& Wood-Robinson, C. (1994). Young People's Understanding of Science Concepts: Implications of Cross-age Studies for Curriculum Planning. Studies in Science Education, vol. 24, n. 1, 75-100.

Garret, R. M. (1988). Resolución de problemas y creatividad: Implicaciones para el currículum de ciencias. Revista Enseñanza de las Ciencias, vol. 6, n. 3, 224-230.

Geddis, A. N. (1990). The Tyranny of the "Right Answer": The Systematic Distortion of Scientific Knowledge. Paper presented at the Annual Conference of the Canadian Society for the Study of Education, 1-22.

Genyea, J. (1983). Improving Student's Problem-solving Skills. A Methodological Approach for a Preparatory Chemistry Course. Journal of Chemical Education, vol. 60, n. 3, 478-481.

Giere, R. (1992). Introduction Cognitive Models of Science. Minneapolis: University of Minnesota Press. 
Estudios Pedagógicos XL, $\mathrm{N}^{\circ}$ 2: 283-302, 2014

¿QUÉ PIENSAN LOS PROFESORES DE QUÍMICA EN EJERCICIO ACERCA DE LA RESOLUCIÓN DE PROBLEMAS CIENTÍFICOS ESCOLARES Y SOBRE LAS COMPETENCIAS DE PENSAMIENTO CIENTIIFICO?

Gil-Perez, D., Dumas-Carr, A., Caillot, M. \& Martinez-Torregrosa, J. (1990). Paper and Pencil Problem Solving in the Physical Science as a Research Activity. Studies in Science Education, vol. 18, n. 1, 137-151.

Henao, B. \& Stipcich, M. (2008). Science Education and Argument: The Prospect of Toulmin as a Possible Response to Contemporary Demands and Challenges for the Teaching of Experimental Sciences. Revista Electrónica Enseñanza de las Ciencias, vol. 7, n. 1, 47-62.

Huffman, D. (1997). Effect of Explicit Problem Solving Instruction on High School Students' Problem-solving Performance and Conceptual Understanding of Physics. Journal of Research in Science Teaching, vol. 34, n. 6, 551-570.

Izquierdo-Aymerich, M., Merino, C. y Quintanilla, M. (2014). La re-construcción de un 'hecho de vida' para enseñar a decidir... y a argumentar en la clase de ciencias. En M. Quintanilla (Comp.), Las competencias de pensamiento científico desde 'las emociones, sonidos y voces' del aula (pp. 31-52). Santiago de Chile: Editorial Bellaterra.

(2004). Un nuevo enfoque de la enseñanza de la química: Contextualizar y modelizar.

The Journal of the Argentine Chemical Society, vol. 92, n. 4-6, 115-136. . \& Adúriz-Bravo, A. (2003). Epistemological Foundations of School Science. Science \& Education, vol. 12, n. 1, 27-43.

Klahr, D. (2002). Exploring Science: The Cognition and Development of Discovery Processes. Massachusetts: MIT Press.

Koponen, I. \& Pehkonen, M. (2010). Coherent Knowledge Structures of Physics Represented as Concept Networks in Teacher Education. Science \& Education, vol. 19, n. 3, 259-282. (2007). Models and Modelling in Physics Education: A Critical Re-analysis of Philosophical Underpinnings and Suggestions for Revisions. Science \& Education, vol. 16, n. 7-8, 751-773.

Labarrere, A. (2012). La solución de problemas, eje del desarrollo del pensamiento y las competencias de pensamiento científico de los estudiantes en matemática y ciencias experimentales. En M. Quintanilla (Comp.), Las competencias de pensamiento científico desde 'las voces' del aula (pp. 15-46). Santiago de Chile: Ediciones Bellaterra.

Molina, N., Arellano, M., Merino, C., Jara, R. \& Quintanilla, M. (2012). Nociones de aprendizaje, enseñanza y competencias de pensamiento científico en profesores de química en formación. $X X V$ Encuentros de Didácticas de las Ciencias Experimentales, Santiago de Compostela, España.

Nurrenbern, S. C. \& Pickering, M. (1987). Concept Learning Versus Problem Solving: Is there a Difference? Journal of Chemical Education, vol. 4, n. 6, 508-510.

Prins, G. T., Bulte, A. M. W., van Driel, J. H. \& Pilot, A. (2008). Selection of Authentic Modeling Practices as Contexts for Chemistry Education. International Journal of Science Education, vol. 30, n. 14, 1867-1890.

Quintanilla, M. (2012). La investigación en evaluación de Competencias de Pensamiento Científico desde la formación continua del profesorado. Algunas directrices epistemológicas. En M. Quintanilla (Comp.), Las competencias de pensamiento científico desde 'las voces' del aula (pp. 1546). Santiago de Chile: Editorial Bellaterra. ., Joglar, C., Jara, R., Camacho, J., Ravanal, E., Labarrere, A. \& Chamizo, J. (2010). School Scientific Problem Solving and Fostering Scientific Thinking Competencies: What Do Inservice Chemistry Teacher Think? Revista Enseñanza de las Ciencias, vol. 28, n. 2, 185-198.

(2006). Identification, Characterization and Evaluation of Scientific Competence from a Naturalized Image of Science. In M. Quintanilla \& A. Adúriz-Bravo (Eds.), Enseñar ciencias en el nuevo milenio. Retos y propuestas (pp. 17-42). Santiago de Chile: Ediciones UC.

Ravanal, E. \& Quintanilla, M. (2010). Characterization of Epistemological View in Biology Teacher on Nature of Science. Revista Electrónica de Enseñanza de las Ciencias, vol. 9, n. 1, 111-124.

Toulmin, S. (1972). Human Understanding. Oxford: Clarendon Press.

Zimmerman, J. \& Forlizzi, J. (2008). The Role of Desing Artifacts in Desing Theory Construction. Artifact, vol. 2, n. 1, 41-45. 